\title{
Coordinated Attention and Resuscitation in Code Blue Events through the CARES System: A Preliminary Evaluation
}

\author{
Luis A. Castro, Luis-Felipe Rodríguez, Adrián Macías, Manuel Domitsu and \\ Moisés Rodríguez \\ Departamento de Computación y Diseño \\ Instituto Tecnológico de Sonora \\ luis.castro@acm.org, luis.rodriguez@itson.edu.mx, adrian.macias@itson.edu.mx, \\ manuel.domitsu@itson.edu.mx, moises.rodriguez@itson.edu.mx
}

\begin{abstract}
Code Blue refers to a medical situation in which a patient suffers cardiac or respiratory arrest and requires immediate cardiopulmonary resuscitation (CPR). A few systems have been developed to support these types of situations. In this paper, we present a preliminary evaluation of CARES, a system that employs mobile technology to support the coordination of response teams in Code Blue emergencies to minimize response times. The evaluation of the CARES system is aimed at exploring the usefulness and ease of use constructs in medical staff. From the data collected, participants agree that the system would be easy to use. Particularly, the Ease of Use factor was rated generally higher that the Usefulness construct by our participants.
\end{abstract}

Keywords: Code Blue Emergency, Medical Informatics, Preliminary evaluation.

\section{Introduction}

When a patient is hospitalized, a major concern of the medical system is to increase the chances of survival among patients suffering cardiopulmonary arrest, which occurs in the order of $10-20 \%[1,2]$. Code Blue refers to a medical situation in which a patient suffers cardiac or respiratory arrest and requires immediate cardiopulmonary resuscitation (CPR). In general, hospitals have a team of trained people to respond to this type of emergency. Particularly, the coordination of such response teams and the time during resuscitation are key aspects to increase the chances of survival among patients in Code Blue situations [1-3]. Code Blue situations require the coordination of an emergency team dedicated to providing cardiopulmonary resuscitation to patients. This type of medical emergency involves the mobilization of human and material resources towards a particular area of a hospital. Additionally, it is necessary the use of cardiopulmonary resuscitation equipment located in the emergency crash cart (also known as red cart or code cart). In many cases, in units where patient care is 
not critical, such as in intermediary care units ${ }^{1}$, nurses or patient's family members notify of Code Blue emergencies when they observe a significant deterioration in the patient's condition. The literature reports that if a patient is treated within the first five minutes, chances of survival are greater and there is less risk of neurological damage $[3,4]$. Since 2000 , there has been significant progress in reducing mortality in cardiac arrests in hospitals. However, there are no structured and flexible systems that allow qualified personnel to respond promptly within hospitals [5].

According to [4], the most common errors in Code Blue emergencies are associated with the communication among response team members and with the algorithms of Advanced Cardiovascular Life Support (ACLS). Moreover, the literature reports that the human factor is essential for timely care in hospitals (e.g., direct supervision of nursing staff) [6]. In this context, in some research fields in which technology is used to address problems related to situations such as Code Blue emergencies, some research lines are focused on studying the automated monitoring of patients [7, 8] and the development of infrastructure to facilitate the communication among mobile nodes [9]. The literature also reports work to support the coordination of emergency teams [10-12]. For example, in [12] the authors designed a simulation to improve the coordination of firefighters. In [10], it is presented a model based on information technologies for crisis management. However, to the best of the authors' knowledge, there are no systems or models designed to facilitate the coordination of response teams in Code Blue emergencies. As mentioned above, this situation requires finding the location and the selection of human resources and the availability of cardiopulmonary resuscitation equipment and personnel.

This paper presents a preliminary evaluation of CARES (Coordinated Attention and Resuscitation in Emergency Situations), a system that employs mobile technology to support the coordination of emergency teams in Code Blue situations. The primary goal of the system is to minimize response times in attending Code Blue situations by improving the coordination of emergency teams. The evaluation is aimed at exploring the usefulness and ease of use constructs in medical staff.

\subsection{The CARES System}

The CARES system consists of four modules: Code Blue Event, Location, Coordination, and Notification modules (Figure 1). Once the Code Blue Event module initiates the process, the Location Module locates qualified personnel and material resources available for responding to the Code Blue emergency. Afterwards, using the output information from the Location Module, the Coordination Module selects the appropriate medical personnel to respond to the Code Blue emergency based on criteria such as their availability, the roles they can take, and their physical proximity to the event location. Finally, the Notification Module notifies the medical personnel required to participate in the Code Blue emergency.

${ }^{1}$ In intensive care units, patients usually are monitored by specialized equipment, besides personnel and family members. 


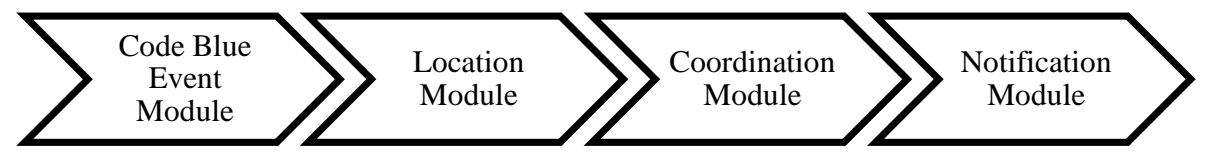

Fig. 1. Conceptual model implemented in CARES

\section{Methods}

We conducted a preliminary evaluation with medical personnel at a local hospital in northwest Mexico attending a total of 2,019 towns and villages (339,773 persons).

\subsection{Participants}

The participants of the evaluation session were 9 medical staff (Male=2, Female=7). The age of participants was 27 years old in average ( $\mathrm{SD}=9.46$ years), ranging from 22 to 46. Seven of them were interns. All of them were mobile phone users, and computer users. For the recruitment, we contacted the Head of the Training and Teaching department at the aforementioned hospital, who was in charge of inviting interns, residents, physicians and nurses. According to the number of people invited to participate, the turnout rate was around $30 \%$.

\subsection{Evaluation Session}

The evaluation session lasted for about an hour and consisted of the following stages:

- Introduction (10 $\mathrm{min})$ : A 10-min introduction to the project and the research team was given.

- Presentation (20 min): The research team presented the overall goals of the project and the current status of the CARES system.

- Scenario (10 min): A scenario (audio only) was presented to the participants (Table 1).

- Video (5 min): A $2 \mathrm{~m} 24 \mathrm{~s}$ video was presented showing the main functionality of the system, based on the previous scenario.

- Evaluation instrument (10 min): Finally, a survey was answered by the participants based on the presentation, scenario, and video. This instrument consisted of a 16item instrument based on the Technology Acceptance Model (TAM) [13]. The scale of the instrument was a 7-item likert-type scale, ranging from Strongly Agree (value=1) to Strongly Disagree (value=7). The questionnaire took around 5 minutes to complete. 
Table 1. Scenario used during the evaluation session

Don Carlos is 42 years old. He was in the Intensive Care Unit (ICU) due to an acute inferior myocardial infarction, where a percutaneous transluminal coronary angioplasty (PTCA) of the right coronary artery was performed. Afterward, he was relocated to a less intensively monitored unit such as the Intermediate Care Unit where he has been stable, but under frequent medical care. Luisa, a 20-year experience nurse, who is in charge of taking care of Don Carlos observes that he is experiencing tachycardia and, given his clinical record, decides to raise the Code Blue alert through the recently-installed CARES system. The CARES system takes into account the location of nurses and physicians for deciding who are the most proximate for attending the emergency. In addition, the CARES system checks among the personnel those who are qualified for attending the emergence (e.g., experience, training, etc.). Moreover, the CARES system checks the staff's availability at that moment to attend the situation. Through the CARES system, Dr. Gonzalez, a resident, receives an alert in his smart wristband saying that there is an ongoing Code Blue situation. Dr. Gonzalez takes out his mobile phone from his pocket to receive further instructions. In his smartphone, Dr. Gonzalez is told that he will be in charge of taking the crash cart to the 322 bed (Don Carlos's bed), given that he is close to the cart at that moment. Meanwhile, Dr. Lopez, who was very close to Don Carlos's location at that time, is instructed to quickly move to Don Carlos's bed, where he is assigned the role of Team Leader in the emergency response team (ERT). Due to training the Dr. Lopez has previously received, he leads the selected nurses and physicians as they arrive. Luisa, who has been close to Don Carlos, received instructions from Dr. Lopez to be in charge of taking notes (e.g., medication administered, doses, time of day). In the same way, Dr. Bobadilla has been assigned the role of Respiratory (Airway), where she is in charge of managing the airway as directed by the Code Team Leader. After the arrival of Dr. Gonzalez, with the crash cart, the patient was stabilized and stayed in sinus rhythm.

\section{Results}

At this point, the evaluation of the CARES system focused on capturing the perception of potential users in the medical field. Opinion seemed to be divided regarding Q_8 "Using CARES in my job would increase my productivity." Many respondents $(\mathrm{N}=3,33 \%)$ agreed only slightly with the statement. Still, almost an equal number of respondents $(\mathrm{N}=4,44.44 \%)$ said they strongly agree with the statement. Also, opinion seemed divided regarding Q_10 "Using CARES would make it easier to do my job" in which a significant number of participants $(\mathrm{N}=4,44.44 \%)$ strongly agreed with the statement, but two $(\mathrm{N}=2,22.22 \%)$ participants only agreed, and the same number of participants slightly agreed. The same was for Q_13 "I would find it easy to get CARES to do what I want it to do." Still, it is noteworthy that none of the respondent disagreed at any level. 


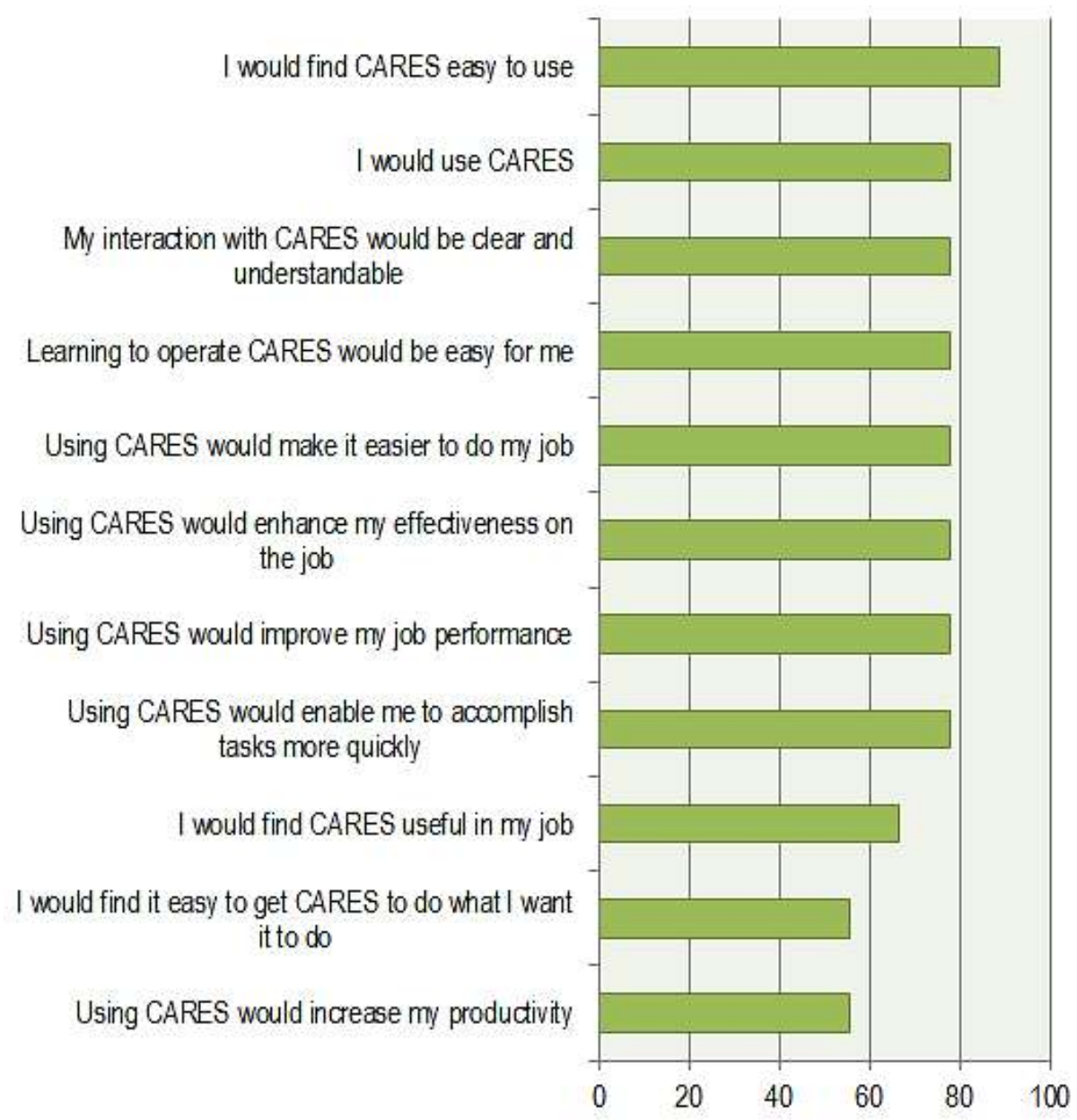

Fig. 2. The CARES system by first rating (strongly agree + agree) and ranked based on highest percentage of respondents' choice

Following Figure 2, it can be seen that the percentage of participants who recorded first rating (strongly agree + agree) was the highest for the item "I would find CARES easy to use", and the least for the item "Using CARES would increase my productivity". This means that our participants generally perceived the CARES system as easy to use, but it was difficult for them to foresee any increase in their productivity through system usage. As show in Figure 2, the items which were endorsed with first order ratings are related to the perceived ease of use construct, whereas the items related to usefulness, although positive, were generally rated second or third order ratings. 
Luis A. Castro, Luis-Felipe Rodríguez, Adrián Macías, Manuel Domitsu, Moisés Rodríguez.

Table 2. Summary Statistics of the Computed Factors and Reliability estimates per construct

\begin{tabular}{|c|c|c|c|c|c|c|c|}
\hline \multirow{2}{*}{ Construct } & \multirow{2}{*}{$\begin{array}{c}\text { Mean } \\
\text { (SD) }\end{array}$} & \multirow{2}{*}{ Median } & \multirow{2}{*}{ Mode } & \multicolumn{3}{|c|}{ Percentiles } & \multirow{2}{*}{$\begin{array}{c}\text { Cronbach's } \\
\quad \text { Alpha }\end{array}$} \\
\hline & & & & 25 & 50 & 75 & \\
\hline Usefulness & $\begin{array}{c}1.82 \\
(0.23)\end{array}$ & 1.60 & 1 & 1.00 & 1.60 & 2.60 & .953 \\
\hline $\begin{array}{l}\text { Ease of } \\
\text { Use }\end{array}$ & $\begin{array}{c}1.69 \\
(0.25)\end{array}$ & 1.20 & 1 & 1.00 & 1.20 & 2.50 & .891 \\
\hline
\end{tabular}

Table 2 shows descriptive statistics regarding each of the constructs. Please note that those values refer to ordinal values assigned: Strongly agree $=1$, strongly disagree $=7$, meaning that lower ratings are in this case associated with positive endorsements. Notably, the Ease of Use construct received higher ratings than that of Usefulness, and this is reflected in the media and median. From the results obtained, the items related to ease of use, had the lower rating (i.e., strongly agree), following very close those related to effectiveness in their work duties and intention to use the system. The mode for all items was 1, meaning that Strongly Agree was the most common answer for all items.

Although some studies have emphasized the barriers for adoption of information systems in general, we carried out a preliminary evaluation to explore the perception of potential users in adopting the CARES system to help them in code blue emergency events in hospitals.

We found surprising that the Ease of Use factor was rated generally higher that the Usefulness construct. We believe there is an explanation for this, which may be related to an unintended sample bias. When carefully looking at the individual rating and comparing among the different backgrounds, interns generally rated lower those items related to usefulness. For instance, interns rated lower the CARES system in terms of ease of use (media $=1.6, \mathrm{SD}=0.8$ ) as opposed to non-interns (media=1.0, $\mathrm{SD}=0.0$ ). We believe that this may be due to the fact that the interns had very limited experience in code blue events, as reported by them in the survey. Therefore, it was difficult for them to see how they could benefit from using the CARES. The opposite seemed to happen to more experienced staff who chose first ratings generally for all items i.e., they were more optimistic as opposed to interns who seemed to be more skeptical or had no idea how the system could benefit them. Another explanation for this can be that the latter personnel are mainly devoted to administrative rather than operative duties like the interns.

It can be argued that this perception could change with experience as it has been reported that text-based communication systems in hospitals have improved efficiency [14]. On the other hand, other studies have evaluated usage and user perceptions on smartphone systems [15-17]. Each work reported positive outcomes and demonstrated user uptake and usage of the intervention during the study period. However, a deeper study is required to explore this issue.

Internal reliability was generally higher but there may be certain items that were not rated as high. Looking retrospectively, the writing of some of the items might not 
have been as straightforward as the others, and that could have been one of the reasons for the variance in their answers.

\section{Conclusions and Future Work}

From the data collected, participants seem to agree that the system would be easy to use. However, they were less convinced that the CARES system would be indeed useful in the workplaces. This study had several limitations, including the small number of participants, mainly interns. The age/experience difference could offer greater insights into this variation, but further studies are needed to corroborate this. For instance, having a greater sample from various hospitals could help consolidate these findings. Also, conducting qualitative studies such as semi-structured interviews could help better understand these results. Also, the CARES systems needs to address ethical concerns related to in-hospital practices, which certainly require careful consideration before a system like CARES can be implemented.

\section{Acknowledgements}

This work was funded by the Instituto Tecnológico de Sonora under grant No. PROFAPI_00302. We thank the medical personnel who participated in the evaluation as well as students who worked on this project.

\section{References}

1. Schultz, S.C., et al., Predicting in-hospital mortality during cardiopulmonary resuscitation. Resuscitation, 1996. 33(1): p. 13-17.

2. McGrath, R., In-house cardiopulmonary resuscitation--after a quarter of a century. Annals of emergency medicine, 1987. 16(12): p. 1365-8.

3. Chan, P.S., et al., Delayed Time to Defibrillation after In-Hospital Cardiac Arrest. New England Journal of Medicine, 2008. 358(1): p. 9-17.

4. Hunt, E.A., et al., Simulation of In-Hospital Pediatric Medical Emergencies and Cardiopulmonary Arrests: Highlighting the Importance of the First 5 Minutes. Pediatrics, 2008. 121(1): p. e34-e43.

5. Lopez Messa, J.B., Paro cardiaco hospitalario: Señales de cambio. Medicina Intensiva, 2010. 34(3): p. 159-160.

6. Jones, D., et al., Circadian pattern of activation of the medical emergency team in a teaching hospital. Critical Care, 2005. 9(4): p. R303-R306.

7. Ko, J., et al., MEDiSN: Medical emergency detection in sensor networks. ACM Transactions on Embedded Computing Systems, 2010. 10(1): p. 1-29.

8. Shnayder, V., et al., Sensor Networks for Medical Care, 2005, Division of Engineering and Applied Sciences, Harvard University.

9. Malan, D., et al. CodeBlue: An Ad Hoc Sensor Network Infrastructure for Emergency Medical Care. in Workshop on Applications of Mobile Embedded Systems (In conjunction with ACM/USENIX Mobisys 2004). 2004. Boston, MA. 
10.Shen, S.Y. and M.J. Shaw. Managing Coordination in Emergency Response Systems with Information Technologies. in Proc. of the 10th Americas Conference on Information Systems (AMCIS 2004). 2004. New York, New York.

11.Hiltz, S.R., P. Diaz, and G. Mark, Introduction: Social media and collaborative systems for crisis management. ACM Transactions on Computer-Human Interaction, 2011. 18(4): p. 1 6.

12.Toups, Z.O., A. Kerne, and W.A. Hamilton, The Team Coordination Game: Zero-fidelity simulation abstracted from fire emergency response practice. ACM Transactions on Computer-Human Interaction, 2011. 18(4): p. 1-37.

13.Davis, F.D., Perceived usefulness, perceived ease of use, and user acceptance of information technology. MIS quarterly, 1989: p. 319-340.

14.Wu, R., et al., Short message service or disService: Issues with text messaging in a complex medical environment. International journal of medical informatics, 2014. 83(4): p. 278-284.

15.O'Connor, C., et al., The use of wireless e-mail to improve healthcare team communication. Journal of the American Medical Informatics Association, 2009. 16(5): p. 705-713.

16.Wu, R.C., et al., The use of smartphones for clinical communication on internal medicine wards. Journal of Hospital Medicine, 2010. 5(9): p. 553-559.

17.Hansen, T.R. and J.E. Bardram, Applying mobile and pervasive computer technology to enhance coordination of work in a surgical ward. Studies in health technology and informatics, 2007. 129(1): p. 107. 